MZ-TH//96-31

hep-th/9610128

\title{
On knots in subdivergent diagrams
}

\author{
Dirk Kreimer円 \\ Dept. of Physics \\ Univ. of Mainz \\ Staudingerweg 7 \\ 55099 Mainz \\ Germany
}

\begin{abstract}
We investigate Feynman diagrams which are calculable in terms of generalized one-loop functions, and explore how the presence or absence of transcendentals in their counterterms reflects the entanglement of link diagrams constructed from them and explains unexpected relations between them.
\end{abstract}

\section{Introduction}

Recently, a connection between knot theory and renormalization theory emerged. It initiated new results in field theory and number theory, via the identification of knots extracted from the topology of Feynman diagrams, with transcendentals found in their overall divergent contributions [1, 2, 3, 4, , 5, 6, 7, 8, 9, 10, 11]. The idea explored in these papers can be described as follows. We consider an overall divergent Feynman diagram. It is well known that after proper renormalization of its subdivergences, its overall divergence will depend on external parameters like masses and momenta only in a trivial (polynomial) manner. It further depends on the spin representation of the involved particles, and on the topology of the diagram in a nontrivial manner. With the spin of the particles specified, one can thus expect to find topological information in the values of the overall divergences. We will now shortly summarize the results achieved so far, and explain how the present paper fits into this context.

The simplest topologies are provided by ladder diagrams. In [1, 12], the reader will find that such diagrams provide only rational contributions in their overall divergences. Correspondingly, these diagrams are knotfree, when mapped to link diagrams. This mapping is achieved by consideration of the momentum flow in the diagram [1, 3]. Each closed loop momentum contributes a component of a link diagram. The mutual entanglement of these components results from the resolution of vertices into over- and undercrossings, and from the topology of the diagram.

Using this approach, it is shown in [1] that the transcendentals $\zeta(2 l-3)$ (odd zetas) appear in $l$-loop Feynman diagrams which deliver $(2,2 l-3)$ torus knots. These are the simplest positive knots, and they are

\footnotetext{
${ }^{1}$ supported by a Heisenberg Fellowship email: kreimer@dipmza.physik.uni-mainz.de
} 
generated by Feynman diagrams which are distinguished from the ladder topology by only one propagator, which crosses all the other rungs.

Up to the five-loop level, only these knots appear, and correspondingly, all transcendentals in counterterms are of odd zeta type. In [3], it is demonstrated that a certain six-loop graph generates a different knot, with eight crossings. This is a success for knot theory: precisely such graphs deliver for the first time a new transcendental, a double sum of weight eight, which was first observed by David Broadhurst [13].

Such multiple sums are known in the literature as Euler/Zagier sums [2, 8, 11, 14]. The restricted class of non-alternating sums is known as multiple zeta values (MZVs) [11.

In [A], these lines of thinking were extended to the seven-loop level, and the scheme independent contribution to the seven-loop $\beta$-function of $\phi^{4}$ theory was calculated. This involved only diagrams free of subdivergences. Again, the match between knots and transcendentals was striking, and culminated in the identification of the first irreducible triple sum of weight eleven with the unique positive four-braid knot.

In [2], Broadhurst enumerates alternating sums (Euler/Zagier sums), inspired by knot and field theory. The results inform number theory, by answering the question how many of such transcendentals are independent over the rational numbers. Further results appeared in [8].

In [6], we use the $\epsilon$-expansion of critical exponents to enlargen our knot-to-number dictionary. This implicitly incorporates graphs with subdivergences via large $N$ methods.

In [7], we identify whole classes of positive knots with MZVs up to 15 crossings, and conjecture the enumeration of irreducible MZVs. This conjecture is further confirmed in 11 .

In [9], an explanation for the connection between knot theory and renormalization theory is given, by indicating how the overall divergences of Feynman diagrams (restricted to diagrams free of subdivergences) provide a weight system, and thus are related to knot invariants of finite type. The investigations are restricted to the study of graphs without subdivergences.

In [10], examples at the four-and five-loop level confirm these findings.

When one carefully studies the above papers, one notices that they barely discuss graphs with subdivergences. In this paper, we want to close the gap. It is the purpose of this paper to compare the UV-divergent behaviour of Feynman graphs which have subdivergences with the behaviour of link and knot diagrams associated to these diagrams.

In the before-mentioned papers the topologies of the diagrams were complicated, but in most cases free of subdivergences. Now, we focus on fairly simple topologies with subdivergences. From [1], we know one result concerning graphs with subdivergences: that simple topologies, whose forest structure is strictly nested or purely overlapping, provide only rational numbers in their overall divergences. These results are confirmed by independent methods in 12]. Here, we generalize such cases by dressing internal propagators with simple rainbow diagrams which generate disjoint subdivergences.

We consider in this paper Feynman graphs which are calculable in terms of $G$-functions [15]. As a specific example we consider vertex functions at zero momentum transfer (zmt) in Yukawa theory, with fermions coupling to a massless scalar field. We are thus restricted to the study of nested divergences as a starting point. From the results in [1], and from the general structure of renormalization theory, we know that the entanglement of subdivergences for the overlapping case follows a similar pattern, only that one has to sum over all maximal forests of these overlapping divergences. Thus, the combinations of $G$-functions generated by overlapping divergences is ultimately a sum of the combinations which arise from nested divergences, in full accord with the forest formula [16]. This was the motivation to choose vertex-functions as an appropriate testing ground to explore the connection between renormalization and link diagrams in the context of graphs with subdivergences.

The sole purpose of these vertex functions is to serve as a visualization for the combinations of $G$-functions which we want to consider. The only transcendentals which appear in $G$-functions stem from the $\zeta$-function evaluated at integer argument

$$
\Gamma(1-z)=e^{\gamma z} \exp \left(\sum_{j=2}^{\infty} \frac{\zeta(j) z^{j}}{j}\right) .
$$

The only knots we expect to see are $(2, q)$ torus knots, according to the identification of $\zeta(q)$ with these knots in 11, 3, 4. 


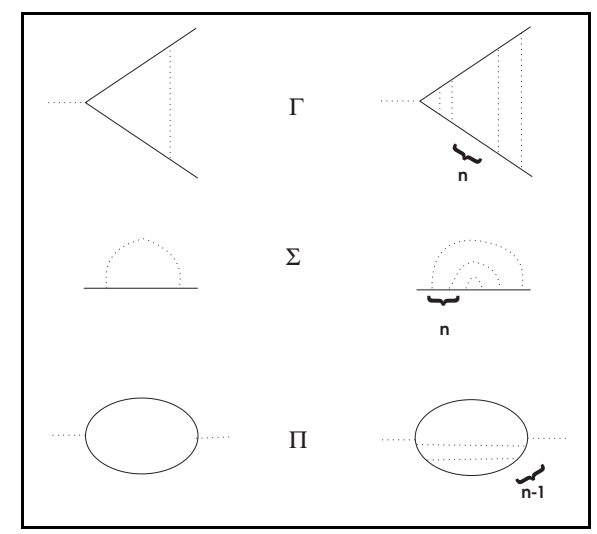

Figure 1: Basic definitions for one-loop functions $\Gamma^{[1]}, \Sigma^{[1]}, \Pi^{[1]}$, on the lhs, and their generalization to $n$-loop functions $\Gamma^{[n]}, \Sigma^{[n]}, \Pi^{[n]}$, on the rhs. Dashed lines indicate the massless spin-0 boson, while the solid line represents a massless fermion.

\section{Definitions}

Our input is a set of generalized one-loop functions. We define $\mathrm{y}^{2}$

$$
\begin{aligned}
G\left(j_{1}, j_{2}\right)\left[q^{2}\right] \equiv & \int \frac{d^{D} k}{N} \frac{1}{\left[(k+q)^{2}\right]^{1+j_{1} \epsilon}\left[k^{2}\right]^{1+j_{2} \epsilon}} \\
:= & {\left[q^{2}\right]^{-\left(j_{1}+j_{2}+1\right) \epsilon} \times } \\
& \times \frac{\Gamma\left[1+\left(j_{1}+j_{2}+1\right) \epsilon\right] \Gamma\left[1-\left(j_{1}+1\right) \epsilon\right] \Gamma\left[\left(1-\left(j_{2}+1\right) \epsilon\right)\right]}{\left(\left(j_{1}+j_{2}+1\right) \epsilon\right) \Gamma\left[\left(2-\left(j_{1}+j_{2}+2\right) \epsilon\right)\right] \Gamma\left[\left(1+j_{1} \epsilon\right)\right] \Gamma\left[\left(1+j_{2} \epsilon\right)\right]},
\end{aligned}
$$

where $D=4-2 \epsilon$ and the last equation fixes the normalization. In this notation, the one-loop radiative corrections of the vertex, the fermion and the scalar propagator in massless Yukawa theory become

$$
\begin{aligned}
\Gamma^{[1]}(q) & =G(0,0)\left[q^{2}\right], \\
\Sigma^{[1]}(q) & =-\frac{1}{2} \not q G(0,0)\left[q^{2}\right], \\
\Pi^{[1]}(q) & =2 q^{2} G(0,0)\left[q^{2}\right],
\end{aligned}
$$

for the (one-loop) vertex-correction at zmt, $\Gamma^{[1]}$; the fermion self-energy, $\Sigma^{[1]}$; and the self-energy of the scalar boson, $\Pi^{[1]}$. These corrections refer to the graphs given in Fig.(11) on the lhs. We can easily generalize this to $n$-loop ladder corrections at the vertex $\Gamma^{[n]}$, and to $n$-loop rainbow corrections for the self-energy of the fermion propagator, $\Sigma^{[n]}$. For the scalar boson, we restrict ourselves to dressings of one internal fermion line, which gives us a function $\Pi^{[n]}$. In Fig.(1) we define these functions diagrammatically on the rhs, and analytically in Eqs.(6.7,8) below.

We denote such diagrams as simple. Such simple topologies deliver rational counterterms, after we absorb the subdivergences by counterterms [1, 12. In short, their overall divergence provides a Laurent series in $(D-4)$ which has coefficients in $\mathbf{Q}$, the rational numbers.

Now these functions are the building blocks for the Feynman diagrams to be considered in this paper. According to the results in [1] we expect a connection to knot theory to appear after renormalization of subdivergences. This is a natural constraint. When the dependence on external parameters is removed via renormalization of subdivergences we shall expect to find an overall divergence which solely reflects the topology of the diagram under consideration.

\footnotetext{
${ }^{2}$ We work in the $\overline{M S}$-scheme, and thus omit irrelevant factors of $\log (4 \pi)$ and $\gamma_{E}$ throughout the paper.
} 
The functions defined in Fig.(1) can be defined analytically by the following iterations

$$
\begin{aligned}
\Gamma^{[n]}(q):= & \int d^{D} k \Gamma^{[n-1]}(k) \frac{1}{\not k} \frac{1}{\not k} \frac{1}{(k+q)^{2}}, \\
\Sigma^{[n]}(q):= & \int d^{D} k \frac{1}{\not k} \Sigma^{[n-1]}(k) \frac{1}{\not k} \frac{1}{(k+q)^{2}}, \\
\Pi^{[n]}(q):= & \operatorname{Tr}\left(\int d^{D} k \frac{1}{\not k} \Sigma^{[n-1]}(k) \frac{1}{\not k} \frac{1}{\not k+\not k}\right), \\
& \Gamma^{[0]}(k):=1, \quad \Sigma^{[0]}(k):=\not k .
\end{aligned}
$$

Using

$$
P_{n} \equiv P_{n}(\epsilon):=\prod_{i:=0}^{n-1} G(0, i)\left[q^{2}=1\right]
$$

one obtains by a standard calculation the following explicit expressions

$$
\begin{aligned}
\Gamma^{[n]}(q) & =\left[q^{2}\right]^{-n \epsilon} P_{n} \\
\Sigma^{[n]}(q) & =\left(-\frac{1}{2}\right)^{n} \not\left[q^{2}\right]^{-n \epsilon} P_{n} \\
\Pi^{[n]}(q) & =2\left(-\frac{1}{2}\right)^{n-1} q^{2}\left[q^{2}\right]^{-n \epsilon} P_{n} .
\end{aligned}
$$

These Green functions are unrenormalized. For $n>1$, they contain subdivergences. To find their overall counterterms we have to renormalize these subdivergences first. For $n>1$ we incorporate the subtraction of subdivergences as follows:

$$
\begin{aligned}
& \Gamma^{[n]} \rightarrow \bar{\Gamma}^{[n]}:=\Gamma^{[n]}-\sum_{i:=1}^{n-1} Z_{\Gamma}^{[i]} \Gamma^{[n-i]}, \\
& Z_{\Gamma}^{[n]}:=<\bar{\Gamma}^{[n]}>, Z_{\Gamma}^{[1]} \equiv<\Gamma^{[1]}>, \\
& \Sigma^{[n]} \rightarrow \bar{\Sigma}^{[n]}:=\Sigma^{[n]}-\sum_{i=1}^{n-1} Z_{\Sigma}^{[i]} \Sigma^{[n-i]}, \\
& Z_{\Sigma}^{[n]}:=<\bar{\Sigma}^{[n]} / \not \mathcal{}>, Z_{\Sigma}^{[1]} \equiv<\Sigma^{[1]} / \not \phi>, \\
& \Pi^{[n]} \rightarrow \bar{\Pi}^{[n]}:=\Pi^{[n]}-\sum_{i:=1}^{n-1} Z_{\Sigma}^{[i]} \Pi^{[n-i]}, \\
& Z_{\Pi}^{[n]}:=<\bar{\Pi}^{[n]} / q^{2}>, Z_{\Pi}^{[1]} \equiv<\Pi^{[1]} / q^{2}>.
\end{aligned}
$$

In angle brackets $\langle\ldots\rangle$ we project onto the proper pole part of the Laurent series in $\epsilon$ and evaluate at $q^{2}=1$. The renormalization of subdivergences is achieved in the $\overline{M S}$-scheme. We introduced renormalization $Z$-factors $Z_{\Gamma \Sigma, \Pi}^{[n]}$ taylored for our purposes. They remove the overall divergence at the indicated loop order in our simple Green functions. These $Z$-factores are totally expressible in terms of the $P_{n}$ functions, for example

$$
Z_{\Gamma}^{[3]}=<P_{3}-\overbrace{<P_{1}>}^{Z_{\Gamma}^{[1]}} P_{2}-\overbrace{<P_{2}-<P_{1}>P_{1}>}^{Z_{\Gamma}^{[2]}} P_{1}>.
$$

Renormalized Green functions (usually referred to by bold letters) are obtained by subtracting the remaining overall divergences

$$
\begin{aligned}
\Upsilon^{[n]}:= & \bar{\Upsilon}^{[n]}-<\bar{\Upsilon}^{[n]}>, \\
& \Upsilon \in\{\Gamma, \Sigma, \Pi\} .
\end{aligned}
$$




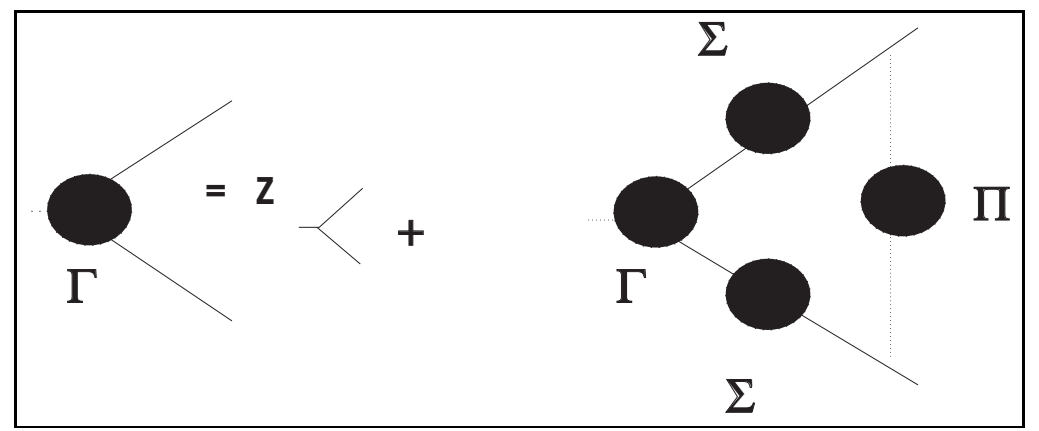

Figure 2: Feynman diagrams are generated by the Schwinger-Dyson equation for the vertex as indicated in the figure. We allow for the selfenergy insertions $\Sigma, \Pi$ defined in the text.

To go from self-energies to propagators, we use

$$
\begin{aligned}
\Delta_{F} & :=\frac{i}{q^{2}-\Pi}, \\
S_{F} & :=\frac{i}{q-\Sigma}, \\
\Pi & :=\sum_{n:=1}^{\infty} \Pi^{[n]}, \\
\Sigma & :=\sum_{n:=1}^{\infty} \Sigma^{[n]} .
\end{aligned}
$$

Quite often we only want to use a terminating geometric series for self-energies with rainbows of a fixed loop number $i$, expanded to a series of degree $k$, so that we define

$$
\begin{aligned}
\Delta_{F}^{[i, k]}(q) & :=\frac{i}{q^{2}}\left[\frac{\Pi^{[i]}(q)}{q^{2}}\right]^{k}, \\
S_{F}^{[i, k]}(q) & :=\frac{i}{\not}\left[\frac{\Sigma^{[i]}(q)}{\not q}\right]^{k} .
\end{aligned}
$$

The corresponding renormalized quantities $\boldsymbol{\Delta}_{\mathbf{F}}{ }^{[i, k]}$ and $\mathbf{S}_{\mathbf{F}}{ }^{[i, k]}$ are obtained by replacing the self-energies $\Sigma^{[i]}, \Pi^{[i]}$ by the corresponding renormalized $\Sigma^{[i]}, \Pi^{[i]}$.

Having defined all these quantities, we now consider the diagrams of Fig.(2). Their generic structure is

$$
\Gamma \sim \int\left[S_{F}^{2} \Delta_{F}\right]^{r}
$$

They come from dressing internal propagators and iterating the vertex as given by the reduced Schwinger Dyson equation of Fig.(2). Propagator dressed with disjoint subdivergences are obtained from expanding the one-particle irreducible functions defined above in a power series in the selfenergies, using Eqs. (26,227).

We investigate the contribution of the so-iterated vertex to the MS $Z$-factor. Especially, we are interested to what extent transcendentals remain after the renormalization of subdivergences. So, in contrast to the simple case, we expect coefficients to be $\notin \mathbf{Q}$. Especially, we expect to see the transcendentals $\zeta(2 l+1)$, generated from Eq.(11) and corresponding to the $(2,2 l+1)$ torus knots, familiar from previous results [1, 3. [. We once more stress that for the case of simple topologies, with undressed internal propagators as in Fig.(1) on the rhs, all transcendentals vanish after the renormalization of proper subdivergences. 


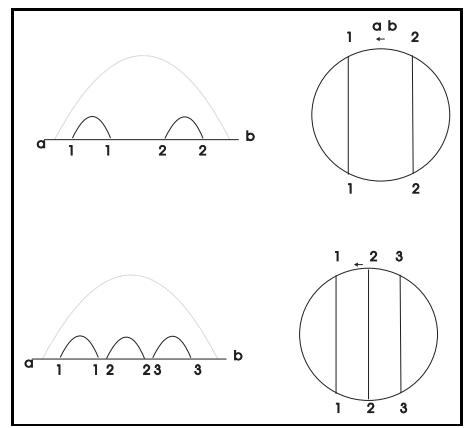

Figure 3: The most basic dressings are topologically equivalent to ladder diagrams. Three selfenergy insertions are needed to get a topology different from a ladder topology. The topology can be encoded as a (Gauss-)code, by reading off sequences like $\{1,1,2,2,3,3\}$ for the case of three disjoint subdivergences, and $\{1,2,3,3,2,1\}$ in the four-loop ladder case.

The most basic example appears when we start to dress $\Gamma^{[1]}$ with one-loop self-energies $\Sigma^{[1]}$ or $\Pi^{[1]}$. As long as there are only one or two such dressings, the topology of the resulting Feynman diagrams is still similar to a ladder topology, as Fig.(3) exhibits. We thus expect trancendentals to cancel, as we claim that there appearance reflects a change from the ladder topology. Indeed, the next section will reveal that transcendentals only remain in case of more than two disjoint subdivergences.

\section{An elementary example}

We start with the consideration of Feynman graphs before the subtraction of subdivergences, with $n$ one-loop bubbles at the fermion line and $m$ one-loop insertions at the boson line, for different $n$ and $m$, but with $n+m=l$ fixed.

To compare cases with a varying number of fermion- or boson self-energies, from now on we normalize Green functions by removing the factors of 2 and $(-1 / 2)$ in Eqs.(12,13) for the selfenergies. In so doing, we employ the fact that $Z_{\Sigma}^{[n]}$ and $Z_{\Pi}^{[n]}$ are related by such simple factors, according to Eqs.(12, 13). After such a normalization (which removes the burden to list superflous factors of 2 in our tables) we have $Z_{\Gamma}^{[n]}=Z_{\Sigma}^{[n]}=Z_{\Pi}^{[n]}=: Z^{[n]}$. This normalization is included in all the results in the tables and adopted in all what follows.

We will consider a function $\Gamma(n, m)$ defined as

$$
\begin{aligned}
\Gamma(n, m)(q) & :=\int d^{D} k \frac{1}{\not k} S_{F}^{[1, n]}(k) \Delta_{F}^{[1, m]}(k+q) \\
& =[G(0,0)]^{n+m} G(0, n+m)\left[q^{2}\right]^{-(n+m+1) \epsilon} .
\end{aligned}
$$

Renormalizing the subdivergences delivers

$$
\begin{aligned}
\bar{\Gamma}(n, m)(q) & :=\int d^{D} k \frac{1}{\not k} \mathbf{S}_{\mathbf{F}}^{[1, n]}(k) \boldsymbol{\Delta}_{\mathbf{F}}{ }^{[1, m]}(k+q) \\
& =\sum_{i=0}^{n+m}\left(\begin{array}{c}
n+m \\
i
\end{array}\right) G(0,0)^{i}[-<G(0,0)>]^{n+m-i} G(0, i)\left[q^{2}\right]^{-(i+1) \epsilon} .
\end{aligned}
$$

The corresponding Feynman graphs are given in Fig.(4). We easily evaluate them using Eqs.(12,13). We are not so much interested in an all order result, but rather compare the results before and after subtraction of subdivergences. 


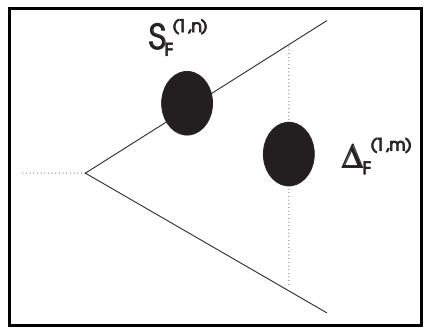

Figure 4: $\bar{\Gamma}(n, m)$.

Table(1) shows that the graphs in general differ for different $n, m$. A few words about the conventions in the tables are in order. Only divergent parts of Green functions are given. The notation $\bar{\Gamma}$ refers to Green functions with subtracted subdivergences. Thus, all the dependence on $q^{2}$ has dropped out. Only in Table(1) we give for comparison a few cases for unsubtracted Green-functions, in the first six entries. For these entries, we have set $q^{2}=1$. Now let us consider Table(1). While still $\Gamma(1,1)=\Gamma(2,0)$, we have $\Gamma(2,1) \neq \Gamma(3,0)$ and $\Gamma(5,1) \neq \Gamma(4,2)$, cf. Table(1) . $^{3}$ Further calculations confirm that in general $\Gamma(n, m) \neq \Gamma\left(n^{\prime}, m^{\prime}\right)$ for $n+m \geq 3$, where we always have $n+m=n^{\prime}+m^{\prime}$.

Now consider the graph after subtraction of subdivergences. A symmetry is emerging. The results only depend on the sum $l=n+m$. Table(1) shows results up to the eleven loop level, given as $\bar{\Gamma}(l)$. The pattern indicated in the table continues to higher loop levels, as checked by systematic calculations. Note that the results in Table(1) are given in a normalization which makes the observed symmetry most obvious. In this normalization we simply have dropped irrelevant factors of 2 or $1 / 2$ which come from the calculation of selfenergies according to Eqs.(12,13,26,27). As a result our Feynman diagrams now solely indicate the nesting of $G$-functions. With this normalization, all the basic one-loop functions have the same divergence.

With these conventions in place, the explicit formulae for $\langle\bar{\Gamma}(2,1)\rangle=\langle\bar{\Gamma}(3,0)\rangle$ are as follows:

$$
\begin{aligned}
<\bar{\Gamma}(2,1)>= & <G(0,0)^{3} G(2,1)-G(0,0)^{2} G(2,0) Z^{[1]} \\
& -2 G(0,0)^{2} G(1,1) Z^{[1]}+3 G(0,0) G(1,0)\left[Z^{[1]}\right]^{2} \\
& -G(0,0)\left[Z^{[1]}\right]^{3}>, \\
<\bar{\Gamma}(3,0)>= & <G(0,0)^{3} G(3,0)-3 G(0,0)^{2} G(2,0) Z^{[1]}+3 G(0,0) G(1,0)\left[Z^{[1]}\right]^{2} \\
& -G(0,0)\left[Z^{[1]}\right]^{3}>,
\end{aligned}
$$

where we used $G(1,0)=G(0,1)$. In both equations, the first term on the rhs is the bare Green function and the other terms renormalize the subdivergences.

As in [1], we now encode the momentum flow of the diagrams into link diagrams, associated to the Feynman diagrams. In this transition, the underlying one-loop vertex-function is simply represented by a circle, and all information as to where external particles couple is lost. Thus, the observed symmetry is obvious, as we clearly see in Fig.(3).

In fact, we can also prove this symmetry from basic properties of our Green functions. We will modify the propagator $\Delta_{F}$ in a way which will not change its high energy behaviour. We usef

$$
\Delta_{F}^{[1, r]}(k)=\Delta_{F}^{[1, r-j]}(k)\left(-i k^{2}\right) \Delta_{F}^{[1, j]}(k), \quad \forall j
$$

and define

$$
\nabla_{F}^{[r, j]}(k, q)=\Delta_{F}^{[1, r]}(k+q)-\Delta_{F}^{[1, r-j]}(k+q)\left(-i k^{2}\right) \Delta_{F}^{[1, j]}(k),
$$

where $q$ is the external momentum. For large $k>>q$ the two terms on the rhs have a similar behaviour. Thus, $\nabla_{F}$ has an improved powercounting, and renders the overall logarithmic divergence in $\bar{\Gamma}(n, m)$ finite,

\footnotetext{
${ }^{3}$ In all the tables, expressions like $a / b \epsilon^{(-r)}$ shall be read as $\frac{a}{b} \frac{1}{\epsilon^{r}}$.

${ }^{4}$ And a similar relation for $S_{F}$, if necessary.
} 


\begin{tabular}{l}
\hline$\Gamma(2,0):=\epsilon^{(-1)}(-1 / 2 \zeta(2)+44 / 3)+8 / 3 \epsilon^{(-2)}+1 / 3 \epsilon^{(-3)}$ \\
\hline$\Gamma(1,1):=\epsilon^{(-1)}(-1 / 2 \zeta(2)+44 / 3)+8 / 3 \epsilon^{(-2)}+1 / 3 \epsilon^{(-3)}$ \\
\hline$\Gamma(3,0):=\epsilon^{(-1)}(-59 / 6 \zeta(3)-11 / 2 \zeta(2)+475 / 4)+\epsilon^{(-2)}(-1 / 2 \zeta(2)+79 / 4)+11 / 4 \epsilon^{(-3)}+1 / 4 \epsilon^{(-4)}$ \\
\hline$\Gamma(2,1):=\epsilon^{(-1)}(-83 / 6 \zeta(3)-11 / 2 \zeta(2)+475 / 4)+\epsilon^{(-2)}(-1 / 2 \zeta(2)+79 / 4)+11 / 4 \epsilon^{(-3)}+1 / 4 \epsilon^{(-4)}$ \\
\hline$\Gamma(5,1):=\epsilon^{(-1)}\left(-113767 / 42 \zeta(6)-155308 / 7 \zeta(5)+1087 / 8 \zeta(4) \zeta(2)-66307 / 7 \zeta(4)+299209 / 126 \zeta(3)^{2}+\right.$ \\
$\left.5470 / 3 \zeta(3) \zeta(2)-437600 / 7 \zeta(3)-49 / 48 \zeta(2)^{3}+427 / 2 \zeta(2)^{2}-10608 \zeta(2)+1451904 / 7\right)+$ \\
$\epsilon^{(-2)}\left(-38827 / 35 \zeta(5)-5435 / 7 \zeta(4)+547 / 6 \zeta(3) \zeta(2)-133468 / 21 \zeta(3)+35 / 2 \zeta(2)^{2}-1200 \zeta(2)+177792 / 7\right)+$ \\
$\epsilon^{(-3)}\left(-1087 / 28 \zeta(4)-10940 / 21 \zeta(3)+7 / 8 \zeta(2)^{2}-122 \zeta(2)+21216 / 7\right)+\epsilon^{(-4)}(-547 / 21 \zeta(3)-10 \zeta(2)+$ \\
$2400 / 7)+\epsilon^{(-5)}(-1 / 2 \zeta(2)+244 / 7)+20 / 7 \epsilon^{(-6)}+1 / 7 \epsilon^{(-7)}$ \\
\hline$\Gamma(4,2):=\epsilon^{(-1)}\left(-132667 / 42 \zeta(6)-181348 / 7 \zeta(5)+1339 / 8 \zeta(4) \zeta(2)-81679 / 7 \zeta(4)+452929 / 126 \zeta(3)^{2}+\right.$ \\
$\left.6730 / 3 \quad \zeta(3) \zeta(2)-538400 / 7 \quad \zeta(3)-49 / 48 \quad \zeta(2)^{3}+427 / 2 \zeta(2)^{2}-10608 \zeta(2)+1451904 / 7\right)+$ \\
$\epsilon^{(-2)}\left(-45337 / 35 \zeta(5)-6695 / 7 \zeta(4)+673 / 6 \zeta(3) \zeta(2)-164212 / 21 \zeta(3)+35 / 2 \zeta(2)^{2}-1200 \zeta(2)+177792 / 7\right)+$ \\
$\epsilon^{(-3)}\left(-1339 / 28 \zeta(4)-13460 / 21 \zeta(3)+7 / 8 \zeta(2)^{2}-122 \zeta(2)+21216 / 7\right)+\epsilon^{(-4)}(-673 / 21 \zeta(3)-10 \zeta(2)+$ \\
$2400 / 7)+\epsilon^{(-5)}(-1 / 2 \zeta(2)+244 / 7)+20 / 7 \epsilon^{(-6)}+1 / 7 \epsilon^{(-7)}$
\end{tabular}

$\overline{\bar{\Gamma}}(1):=1 / 2 \epsilon^{(-1)}-1 / 2 \epsilon^{(-2)}$

$\overline{\bar{\Gamma}}(2):=-1 / 3 \epsilon^{(-1)}-1 / 3 \epsilon^{(-2)}+1 / 3 \epsilon^{(-3)}$

$\overline{\bar{\Gamma}}(3):=\epsilon^{(-1)}(-1 / 2 \zeta(3)+1 / 4)+1 / 4 \epsilon^{(-2)}+1 / 4 \epsilon^{(-3)}-1 / 4 \epsilon^{(-4)}$

$\bar{\Gamma}(4):=\epsilon^{(-1)}(3 / 5 \zeta(4)-2 / 5 \zeta(3)-1 / 5)+\epsilon^{(-2)}(2 / 5 \zeta(3)-1 / 5)-1 / 5 \epsilon^{(-3)}-1 / 5 \epsilon^{(-4)}+1 / 5 \epsilon^{(-5)}$

$\overline{\bar{\Gamma}(5):=\epsilon^{(-1)}(-\zeta(5)+1 / 2 \zeta(4)+1 / 3 \zeta(3)+1 / 6)+\epsilon^{(-2)}(-1 / 2 \zeta(4)+1 / 3 \zeta(3)+1 / 6)+\epsilon^{(-3)}(-1 / 3 \zeta(3)+}$ $1 / 6)+1 / 6 \epsilon^{(-4)}+1 / 6 \epsilon^{(-5)}-1 / 6 \epsilon^{(-6)}$

$\overline{\bar{\Gamma}}(7):=\epsilon^{(-1)}\left(-9 / 4 \zeta(7)+5 / 4 \zeta(6)+3 / 4 \zeta(5)-3 / 4 \zeta(4) \zeta(3)+3 / 8 \zeta(4)+1 / 4 \zeta(3)^{2}+1 / 4 \zeta(3)+1 / 8\right)+$ $\epsilon^{(-2)}\left(-5 / 4 \zeta(6)+3 / 4 \zeta(5)+3 / 8 \zeta(4)-1 / 4 \zeta(3)^{2}+1 / 4 \zeta(3)+1 / 8\right)+\epsilon^{(-3)}(-3 / 4 \zeta(5)+3 / 8 \zeta(4)+1 / 4 \zeta(3)+$ $1 / 8)+\epsilon^{(-4)}(-3 / 8 \zeta(4)+1 / 4 \zeta(3)+1 / 8)+\epsilon^{(-5)}(-1 / 4 \zeta(3)+1 / 8)+1 / 8 \epsilon^{(-6)}+1 / 8 \epsilon^{(-7)}-1 / 8 \epsilon^{(-8)}$

$\bar{\Gamma}(10):=\epsilon^{(-1)}(102 / 11 \zeta(10)-170 / 33 \zeta(9)-63 / 22 \zeta(8)+36 / 11 \zeta(7) \zeta(3)-18 / 11 \zeta(7)+30 / 11 \zeta(6) \zeta(4)-$ $20 / 11 \zeta(6) \zeta(3)-10 / 11 \zeta(6)+18 / 11 \zeta(5)^{2}-18 / 11 \zeta(5) \zeta(4)-12 / 11 \zeta(5) \zeta(3)-6 / 11 \zeta(5)-9 / 22 \zeta(4)^{2}+$ $\left.6 / 11 \zeta(4) \zeta(3)^{2}-6 / 11 \zeta(4) \zeta(3)-3 / 11 \zeta(4)-4 / 33 \zeta(3)^{3}-2 / 11 \zeta(3)^{2}-2 / 11 \zeta(3)-1 / 11\right)+\epsilon^{(-2)}(170 / 33 \zeta(9)-$ $63 / 22 \zeta(8)-18 / 11 \zeta(7)+20 / 11 \zeta(6) \zeta(3)-10 / 11 \zeta(6)+18 / 11 \zeta(5) \zeta(4)-12 / 11 \zeta(5) \zeta(3)-6 / 11 \zeta(5)-$ $\left.9 / 22 \zeta(4)^{2}-6 / 11 \zeta(4) \zeta(3)-3 / 11 \zeta(4)+4 / 33 \zeta(3)^{3}-2 / 11 \zeta(3)^{2}-2 / 11 \zeta(3)-1 / 11\right)+\epsilon^{(-3)}(63 / 22 \zeta(8)-$ $18 / 11 \zeta(7)-10 / 11 \zeta(6)+12 / 11 \zeta(5) \zeta(3)-6 / 11 \zeta(5)+9 / 22 \zeta(4)^{2}-6 / 11 \zeta(4) \zeta(3)-3 / 11 \zeta(4)-2 / 11 \zeta(3)^{2}-$ $2 / 11 \zeta(3)-1 / 11)+\epsilon^{(-4)}\left(18 / 11 \zeta(7)-10 / 11 \zeta(6)-6 / 11 \zeta(5)+6 / 11 \zeta(4) \zeta(3)-3 / 11 \zeta(4)-2 / 11 \zeta(3)^{2}-\right.$ $2 / 11 \zeta(3)-1 / 11)+\epsilon^{(-5)}\left(10 / 11 \zeta(6)-6 / 11 \zeta(5)-3 / 11 \zeta(4)+2 / 11 \zeta(3)^{2}-2 / 11 \zeta(3)-1 / 11\right)+\epsilon^{(-6)}(6 / 11 \zeta(5)-$ $3 / 11 \zeta(4)-2 / 11 \zeta(3)-1 / 11)+\epsilon^{(-7)}(3 / 11 \zeta(4)-2 / 11 \zeta(3)-1 / 11)+\epsilon^{(-8)}(2 / 11 \zeta(3)-1 / 11)-1 / 11 \epsilon^{(-9)}-$ $1 / 11 \epsilon^{(-10)}+1 / 11 \epsilon^{(-11)}$

Table 1: $<\Gamma(n, m)>$ for various values of $\left(n_{1}, n_{2}\right)$. After the renormalization of subdivergences we find that the results for $\langle\bar{\Gamma}(n, m)>$ depend only on the sum $l=n+m$, which we give as $\langle\bar{\Gamma}(l)>$. The transcendental $\zeta(l)$ appears at $l+1$ loops. 
when inserted for $\Delta_{F}$. Consequently, we can replace $\Delta_{F}^{[r]}(k+q)$ by $\Delta_{F}^{[r-j]}(k+q) k^{2} \Delta_{F}^{[j]}(k)$ for any $j$, which gives the observed symmetry.

Next, let us see if we can understand the various transcendentals appearing in the counterterms. First, we note that $\bar{\Gamma}(3)$ is the first case which delivers a new topology different from the ladder topology. It is reasonable to expect that this change in topology is reflected in the overall divergence $\langle\bar{\Gamma}(3)>$. And indeed, we find that $<\bar{\Gamma}(3)>$ contains $\zeta(3)$, and thus should be related to the trefoil knot [3].

There are two possible approaches to assign knots to Feynman diagrams. Either, one maps the possible momentum routings in Feynman diagrams to link diagrams [1] or one investigates the Gauss code of the Feynman diagram under consideration [7]. Both approaches are intuitive and serve the purpose to demonstrate the connection between the transcendental numbers obtained from the Feynman diagrams under consideration and low-dimensional topology. Defining these mappings rigorously is beyond the scope of this paper. A more detailled discussion will be in a forthcoming book [18]. For our present purposes we are contend to reproduce the pattern which we observe in Table(1).

Essentially, we want to reproduce the fact that $\bar{\Gamma}(2 n+1)$ delivers the $(2,2 n+1)$ torus knot (corresponding to the appearance of $\zeta(2 n+1)$ in Table(1)) for $n \geq 1$, and explain why $\bar{\Gamma}(2 n)$ only contains $\zeta(2 n-1)$ and relates to the $(2,2 n-1)$ torus knots, $n \geq 2$.

Let us use a method inspired by Gauss codes [7, 17]. In Fig.(5) we indicate how to obtain the codes $\{1,1,2,2, \ldots, n, n\}$ and $\{1,2,2, \ldots, n, n, 1\}$ for $\bar{\Gamma}(n)$. These are the two distinguished possibilities, referring to the fact that we can either start reading the code between two self-energy insertions, or inside such a bubble. Further, whenever we go through the graph we have two possibilities when we come through a subdivergence, passing along one of its two propagators.

Let us make such a choice for each one-loop insertion in a string of $r$ subdivergence and let us map this to a curve with $r$ curls as indicated in Fig.(5), for each such choice. Fig.(5) gives the example of three oneloop insertions, and an arbitrarily chosen paths through them is indicated. For the string $\{1,1, \ldots, n, n\}$ we simply close the curves at both ends, marked as $a$ and $b$ in the figure. Running through this curve reproduces the same code as obtained from the diagram. The curve is a circle with some curl in it, but unknotted, for all $n$. We thus expect to see rational numbers in the results for $\bar{\Gamma}(n)$ for all $n$.

Next consider the string $\{1,2,2, \ldots, n, n, 1\}$. We know already how to map the substring $2,2, \ldots, n, n$ to a piece of a curve (this is similar to the previous case), but we now simply define the mapping of $\{1,2,2, \ldots, n, n, 1\}$ to a closed curve as indicated at the bottom of Fig.(5). We effectively double the strand which runs from $a$ to $b$ on the side which has lesser curl and close the curve by once more identifying the points $a$ and $b$, and the point marked $x$. Each self-energy which is met by the doubled strand produces two extra crossings. Still, passing along the curve and notating the number of each crossing in the order of appearance reproduces the code. This way of doubling a strand is known as a cabling operation in knot theory. This is in accord with an analysis using momentum routings, and with the experience gained in [1].

Let us count. We need at least three subdivergences for the first knot to appear, and only addition of two further subdivergences through which we pass in different manner will generate a knot with two more crossings. The two more subdivergences are needed because we double the strand at the side with lesser curl. So we need to generate more curl on each side to increase the number of crossings in the knot. This is in accordance with the transcendentals found in $\bar{\Gamma}(n)$ for all $n$. In this manner, by distributing the amount of curl on both sides in all posible ways, one generates all transcendentals observed in Table(1).

Products of $\zeta$ 's can be obtained by lifting the factorization properties of the propagator Eq.(34) to knot diagrams, which produces the factor knots corresponding to the products of lower lying transcendentals in Table(1). These expectations are indeed confirmed by explicit calculations, $\bar{\Gamma}(5)$ in Table(1) provides an example for the appearance of $\zeta(5)$ and $\zeta(3)$ in a six-loop example, $\bar{\Gamma}(7)$ provides $\zeta(7), \zeta(5)$ and $\zeta(3)$ and $\bar{\Gamma}(10)$ provides $\zeta(9), \zeta(7), \zeta(5)$ and $\zeta(3)$, as well as the expected products of $\zeta$ 's and rational contributions. There are hints that the presence of even $\zeta$ 's $\left(\zeta(2 n), n>1, \sim \pi^{2 n}\right)$ is related to the differences in writhe between the different strands which participate in the cabling operation. This is currently under investigation. 


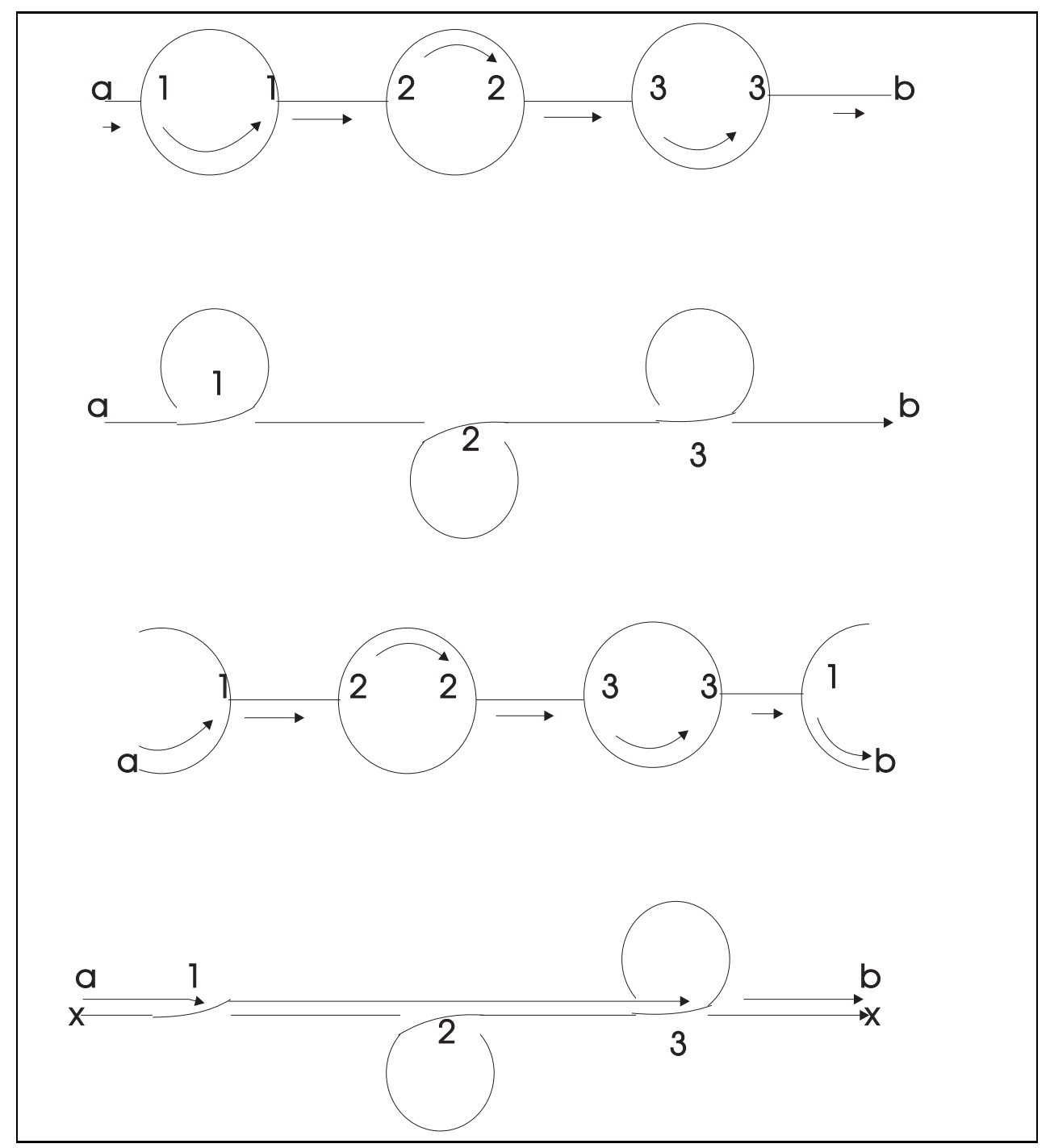

Figure 5: The first line gives a string with three subdivergences. Running through the graph produces a code. This code is obtained from the curve below when we run from $a$ to $b$. There are two principal choices how to run through a one-loop subdivergences, which results in curl on different sides. When we start running in the middle of a subdivergence (third line) we will assign to this a curve which has a doubled strand (fourth line). We double on the side where we have lesser curl, to minimize the number of extra crossings. In the example considered here this is irrelevant as we have one curl on each side. The resulting knot is always a $(2, q)$ torus knot which matches the transcendentals in Table(1). 


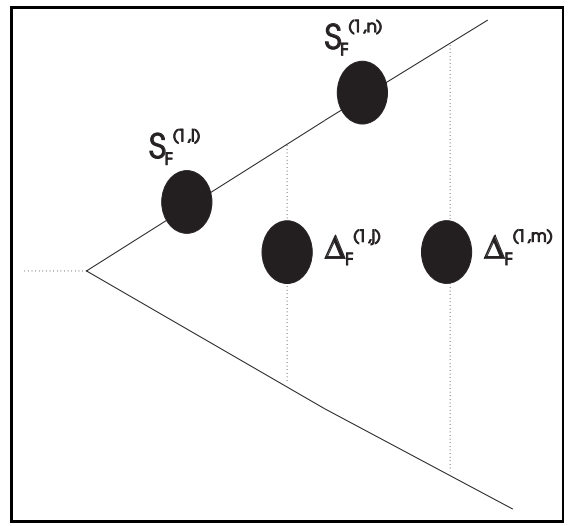

Figure 6: $\bar{\Gamma}(i, j, m, n)$.

\section{Continuation to a dressed two-loop graph}

Our generic topology is indicated in Fig.(6), which we notate by $\bar{\Gamma}(i, j, m, n)$ for a two loop ladder graph with $(i+j+m+n)$ one-loop subdivergences at the indicated places. It is defined by the following expression (this time we omit to mention the case without renormalized subdivergences at all - we know already that all the nice properties will only turn up after renormalization of subgraphs).

$$
\bar{\Gamma}(i, j, n, m)(q):=\int d^{D} k \bar{\Gamma}(i, j)(k) \frac{1}{\not k} \mathbf{S}_{\mathbf{F}}^{[1, n]}(k) \boldsymbol{\Delta}_{\mathbf{F}}{ }^{[1, m]}(k+q) .
$$

In Table(2) we collect results. We spot some remarkable properties which, again, are visible only after elimination of subdivergences.

The case $<\bar{\Gamma}(2,0,0,0)>$ delivers the same value as $\langle\bar{\Gamma}(0,2,0,0)>$. It contains $\zeta(3)$. The symmetry is obvious when studying the analytic expressions for the two cases, from the fact that $G\left(i_{1}, i_{2}\right)=G\left(i_{2}, i_{1}\right)$. As link diagrams, it is obvious that any link diagram for $\bar{\Gamma}(2,0,0,0)$ is a link diagram for $\bar{\Gamma}(0,2,0,0)$ as well. Forgetting the points where external particles couple, the diagrams are topologically equivalent. In contrast, $<\bar{\Gamma}(1,1,0,0)>$ is free of $\zeta(3)$. This is clear from the fact that it is topologically equivalent to a ladder diagram, see Fig.(可), while $<\bar{\Gamma}(2,0,0,0)\rangle$ is topologically equivalent to $<\bar{\Gamma}(3)>$. Fig.(7) demonstrates this clearly. Now a Gauss code analysis for a $n$-loop ladder diagram reveals that all its Gauss codes are equivalent to the code $\{1,1, \ldots, n, n\}$, which explains its rationality in this language.

This points towards an even more striking fact hidden in Table(2) $)$. While $\langle\bar{\Gamma}(2,0,0,0)\rangle=\langle\bar{\Gamma}(0,2,0,0)\rangle$ contain knotted -transcendental- and unknotted -rational- codes, $\langle\bar{\Gamma}(1,1,0,0)>$ contains only the rational code. This is in striking agreement with the fact that the rational contribution for the $(2,0,0,0)$ and $(0,2,0,0)$ cases is the same as the $(1,1,0,0)$ contribution, cf. Table $(2)$.

To test these phenomena, we calculated further examples. Some of them are collected in Table(2). By inspection, we indeed see that the rational parts of

$\{\bar{\Gamma}(2,0,1,1), \bar{\Gamma}(1,1,1,1)\}$,

$\{\bar{\Gamma}(2,0,0,2), \bar{\Gamma}(1,1,0,2)\}$,

$\{\bar{\Gamma}(3,0,0,0), \bar{\Gamma}(2,1,0,0)\}$,

$\{\bar{\Gamma}(3,0,0,1), \bar{\Gamma}(2,1,0,1)\}$,

$\{\bar{\Gamma}(4,0,0,0), \bar{\Gamma}(3,1,0,0)\}$,

$\{\bar{\Gamma}(4,0,0,1), \bar{\Gamma}(3,1,0,1)\}$

are indeed identical, in perfect agreement with the analysis of Gauss codes or link diagrams. . Again, Table(2)

\footnotetext{
${ }^{5}$ We also have the symmetry $(i, j, m, n)=\left(i, j, m^{\prime}, n^{\prime}\right), m+n=m^{\prime}+n^{\prime}$, which one can observe in Table(2). It is similar to the symmetries of $\bar{\Gamma}(n)$.
} 
$\bar{\Gamma}(2,0,0,0):=\epsilon^{(-1)}(1 / 2 \zeta(3)-5 / 12)-1 / 12 \epsilon^{(-2)}+1 / 4 \epsilon^{(-3)}-1 / 12 \epsilon^{(-4)}$

$\overline{\bar{\Gamma}}(1,1,0,0):=-5 / 12 \epsilon^{(-1)}-1 / 12 \epsilon^{(-2)}+1 / 4 \epsilon^{(-3)}-1 / 12 \epsilon^{(-4)}$

$\bar{\Gamma}(0,2,0,0):=\epsilon^{(-1)}(1 / 2 \zeta(3)-5 / 12)-1 / 12 \epsilon^{(-2)}+1 / 4 \epsilon^{(-3)}-1 / 12 \epsilon^{(-4)}$

$\bar{\Gamma}(2,0,0,1):=\epsilon^{(-1)}(-3 / 20 \zeta(4)-1 / 10 \zeta(3))+\epsilon^{(-2)}(-1 / 10 \zeta(3)+19 / 60)-11 / 60 \epsilon^{(-4)}+1 / 15 \epsilon^{(-5)}$

$\overline{\bar{\Gamma}}(1,1,0,1):=19 / 60 \epsilon^{(-2)}-11 / 60 \epsilon^{(-4)}+1 / 15 \epsilon^{(-5)}$

$\overline{\bar{\Gamma}}(0,2,0,1):=\epsilon^{(-1)}(-3 / 20 \zeta(4)-1 / 10 \zeta(3))+\epsilon^{(-2)}(-1 / 10 \zeta(3)+19 / 60)-11 / 60 \epsilon^{(-4)}+1 / 15 \epsilon^{(-5)}$

$\bar{\Gamma}(2,0,0,2):=\epsilon^{(-1)}(3 / 10 \zeta(4)-1 / 5 \zeta(3)+11 / 30)+\epsilon^{(-2)}(1 / 5 \zeta(3)-7 / 45)-19 / 90 \epsilon^{(-3)}+1 / 45 \epsilon^{(-4)}+$ $13 / 90 \epsilon^{(-5)}-1 / 18 \epsilon^{(-6)}$

$\bar{\Gamma}(1,1,0,2):=\epsilon^{(-1)}(-7 / 30 \zeta(5)+1 / 4 \zeta(4)-11 / 30 \zeta(3)+11 / 30)+\epsilon^{(-2)}(-1 / 20 \zeta(4)+1 / 6 \zeta(3)-7 / 45)+$ $\epsilon^{(-3)}(-1 / 30 \zeta(3)-19 / 90)+1 / 45 \epsilon^{(-4)}+13 / 90 \epsilon^{(-5)}-1 / 18 \epsilon^{(-6)}$

$\bar{\Gamma}(2,0,1,1):=\epsilon^{(-1)}(3 / 10 \zeta(4)-1 / 5 \zeta(3)+11 / 30)+\epsilon^{(-2)}(1 / 5 \zeta(3)-7 / 45)-19 / 90 \epsilon^{(-3)}+1 / 45 \epsilon^{(-4)}+$ $13 / 90 \epsilon^{(-5)}-1 / 18 \epsilon^{(-6)}$

$\overline{\bar{\Gamma}}(1,1,1,1):=\epsilon^{(-1)}(-7 / 30 \zeta(5)+1 / 4 \zeta(4)-11 / 30 \zeta(3)+11 / 30)+\epsilon^{(-2)}(-1 / 20 \zeta(4)+1 / 6 \zeta(3)-7 / 45)+$ $\epsilon^{(-3)}(-1 / 30 \zeta(3)-19 / 90)+1 / 45 \epsilon^{(-4)}+13 / 90 \epsilon^{(-5)}-1 / 18 \epsilon^{(-6)}$

$\overline{\bar{\Gamma}}(3,0,0,0):=\epsilon^{(-1)}(-3 / 5 \zeta(4)+1 / 5 \zeta(3)+3 / 10)+\epsilon^{(-2)}(1 / 10 \zeta(3)+1 / 20)+1 / 20 \epsilon^{(-3)}-3 / 20 \epsilon^{(-4)}+$ $1 / 20 \epsilon^{(-5)}$

$\bar{\Gamma}(2,1,0,0):=\epsilon^{(-1)}(-3 / 10 \zeta(4)-2 / 5 \zeta(3)+3 / 10)+\epsilon^{(-2)}(3 / 10 \zeta(3)+1 / 20)+1 / 20 \epsilon^{(-3)}-3 / 20 \epsilon^{(-4)}+$ $1 / 20 \epsilon^{(-5)}$

$\bar{\Gamma}(4,0,0,0):=\epsilon^{(-1)}(\zeta(5)-3 / 10 \zeta(4)-7 / 15 \zeta(3)-7 / 30)+\epsilon^{(-2)}(-1 / 10 \zeta(4)+1 / 5 \zeta(3)-1 / 30)+$ $\epsilon^{(-3)}(-1 / 15 \zeta(3)-1 / 30)-1 / 30 \epsilon^{(-4)}+1 / 10 \epsilon^{(-5)}-1 / 30 \epsilon^{(-6)}$

$\overline{\bar{\Gamma}}(3,1,0,0):=\epsilon^{(-1)}(3 / 10 \zeta(5)+3 / 20 \zeta(4)-17 / 30 \zeta(3)-7 / 30)+\epsilon^{(-2)}(-1 / 4 \zeta(4)+1 / 2 \zeta(3)-1 / 30)+$ $\epsilon^{(-3)}(-1 / 6 \zeta(3)-1 / 30)-1 / 30 \epsilon^{(-4)}+1 / 10 \epsilon^{(-5)}-1 / 30 \epsilon^{(-6)}$

$\bar{\Gamma}(3,0,0,1):=\epsilon^{(-1)}(3 / 20 \zeta(5)-1 / 10 \zeta(4)-4 / 15 \zeta(3)-1 / 12)+\epsilon^{(-2)}(7 / 40 \zeta(4)+11 / 60 \zeta(3)-2 / 15)+$ $\epsilon^{(-3)}(-2 / 15 \zeta(3)-1 / 12)-1 / 120 \epsilon^{(-4)}+7 / 60 \epsilon^{(-5)}-1 / 24 \epsilon^{(-6)}$

$\bar{\Gamma}(2,1,0,1):=\epsilon^{(-1)}(7 / 20 \zeta(5)+1 / 5 \zeta(3)-1 / 12)+\epsilon^{(-2)}(3 / 40 \zeta(4)+1 / 4 \zeta(3)-2 / 15)+\epsilon^{(-3)}(-1 / 5 \zeta(3)-$ $1 / 12)-1 / 120 \epsilon^{(-4)}+7 / 60 \epsilon^{(-5)}-1 / 24 \epsilon^{(-6)}$

$\bar{\Gamma}(4,0,0,1):=\epsilon^{(-1)}\left(-2 / 7 \zeta(6)-3 / 35 \zeta(5)+17 / 70 \quad \zeta(4)-6 / 35 \quad \zeta(3)^{2}-1 / 105 \quad \zeta(3)+1 / 105\right)+$ $\epsilon^{(-2)}(-8 / 35 \zeta(5)-4 / 35 \zeta(4)+38 / 105 \zeta(3)+16 / 105)+\epsilon^{(-3)}(9 / 70 \zeta(4)-29 / 105 \zeta(3)+1 / 105)+$ $\epsilon^{(-4)}(3 / 35 \zeta(3)+11 / 210)+1 / 105 \epsilon^{(-5)}-17 / 210 \epsilon^{(-6)}+1 / 35 \epsilon^{(-7)}$

$\overline{\bar{\Gamma}}(3,1,0,1):=\epsilon^{(-1)}\left(-5 / 14 \zeta(6)+39 / 70 \zeta(5)-1 / 28 \zeta(4)+29 / 70 \zeta(3)^{2}+67 / 210 \zeta(3)+1 / 105\right)+$ $\epsilon^{(-2)}(-3 / 14 \zeta(5)-31 / 140 \zeta(4)+37 / 210 \zeta(3)+16 / 105)+\epsilon^{(-3)}(27 / 140 \zeta(4)-73 / 210 \zeta(3)+1 / 105)+$ $\epsilon^{(-4)}(9 / 70 \zeta(3)+11 / 210)+1 / 105 \epsilon^{(-5)}-17 / 210 \epsilon^{(-6)}+1 / 35 \epsilon^{(-7)}$

Table $2:<\bar{\Gamma}(i, j, m, n)>$ 


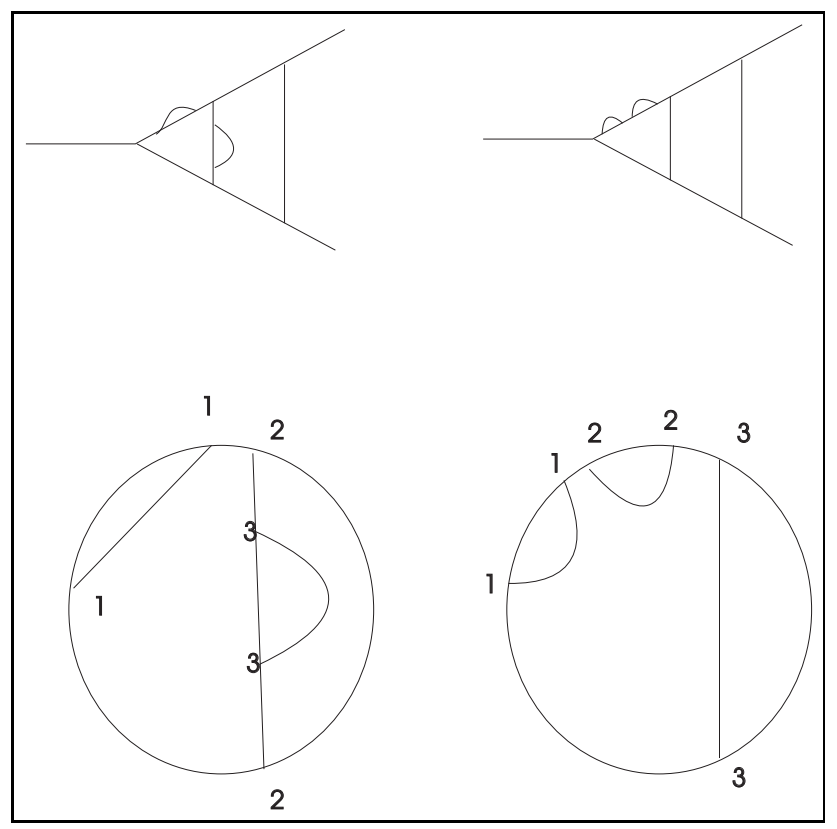

Figure 7: $<\bar{\Gamma}(1,1,0,0)>,<\bar{\Gamma}(2,0,0,0)>$ and their topologies and codes drawn below. $\bar{\Gamma}(1,1,0,0)$ reproduces the ladder topology $\{1,2,3,3,2,1\}$ and $\bar{\Gamma}(2,0,0,0)$ reproduces the same topology as $\bar{\Gamma}(3)$ : $\{1,1,2,2,3,3\}$.

exhibits typical examples, while the reported phenomenon was confirmed systematically to higher orders with results which are too long to be reproduced here.

This indicates new relations between Feynman diagrams, not used and explored so far. Note that these relations are hidden in various ways. First of all, trivial prefactors resulting from various spin factors of the particles hide these relations. For this reason we normalized our graphs omitting the factors in eqs.(12, 13), as mentioned before. These factors seem to play the same role as group theoretic factors in Chern Simons theory, if one likes this analogy, while the knottishness - the topology- is in the transcendentals.

Second, such relations are impossible to observe as long as one does not restrict oneself to the consideration of overall divergences, the leading symbol of the graph, so to speak, by elimination of subdivergences. Again, all symmetries and number theoretic properties are only apparent after proper subtraction of subdivergences, emphasizing the importance of studying proper overall divergences, to see the connection with link theory.

In the next section we mention two further examples, as they are instructive for the reader.

\section{$5 \quad$ Higher order dressing}

We can generalize the examples of the previous section when we increase the number of loops in the ladder, but still have chains of one-loop subdivergences at internal lines. A particularly interesting example is the function $\bar{\Gamma}_{n}(1,0)$, defined in Fig.(8). The figure also explains the rationality of $\bar{\Gamma}_{n}(1,0)$. We refer to the methods of [1, 3] in this case, as a Gauss code is not available. ${ }^{6}$ This rationality agrees with its evaluation in terms of $G$-functions:

$$
\begin{aligned}
& \bar{\Gamma}_{2}(1,0)=-1 / 3 \epsilon^{(-1)}-5 / 24 \epsilon^{(-2)}+1 / 3 \epsilon^{(-3)}-1 / 8 \epsilon^{(-4)}, \\
& \bar{\Gamma}_{3}(1,0)=4 / 15 \epsilon^{(-1)}+13 / 30 \epsilon^{(-2)}-17 / 240 \epsilon^{(-3)}-43 / 240 \epsilon^{(-4)}
\end{aligned}
$$

\footnotetext{
${ }^{6}$ This is due to the fact that there is no closed non-selfintersecting curve which runs through all the vertices of the diagram.
} 


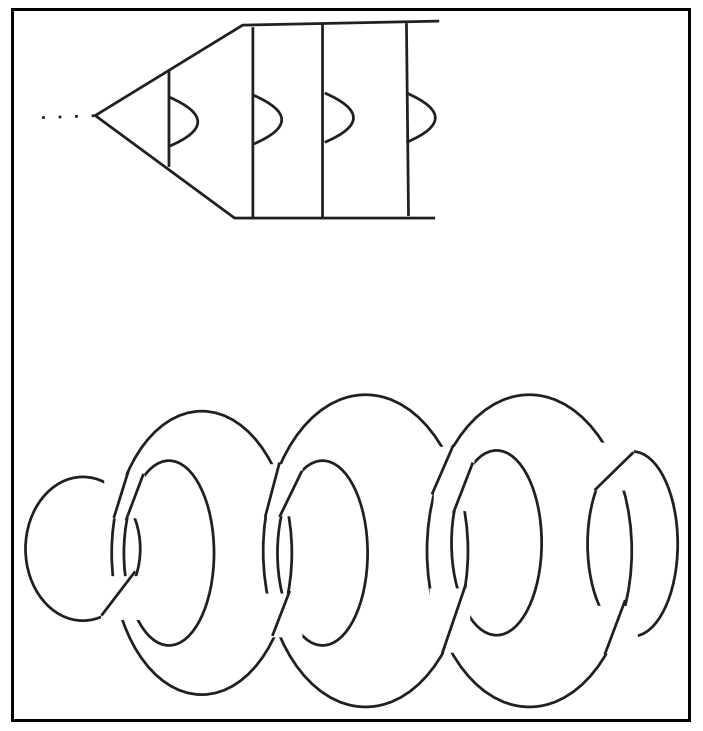

Figure 8: $\bar{\Gamma}_{n}(1,0)$. As a Gauss code is not available in this situation, we indicate the entanglement of a link diagram associated to the graph. The link diagram is knot free.

$$
+5 / 48 \epsilon^{(-5)}-1 / 48 \epsilon^{(-6)} .
$$

We omit to give results for higher loop orders, but again calculations confirm rationality for all cases which have been tested. The cancellation of transcendentals is highly non-trivial, due to the presense of multiple counterterms generated by the subdivergences.

Further, Fig. (9) considers dressings with two-loop rainbows, and shows the appearance of knots matching the transcendentals in the overall divergence, given in Table(3). Note that $\overline{\Gamma_{2}}(2)$ does not provide any knotnumber, but delivers the transcendental $\zeta(4)$, indicating some extra writhe in the diagrams. Further, an analysis with the help of Gauss codes suggest that $\overline{\Gamma_{2}}(2)$ should contain $\zeta(5)$, while $\overline{\Gamma_{2}}(3)$ and $\overline{\Gamma_{2}}(4)$ should have $\zeta(7)$ and $\zeta(9)$, respectively. Which is indeed the case.

\section{Other field theories}

One can extend the results considered here to other renormalizable theories. For clarity, we presented our examples for the case of a massless Yukawa theory. To incorporate other theories, one can resort to the matrix calculus proposed in [i]. This becomes necessary due to the different formfactors which are present in general, resulting from all possible spin structures. One eventually ends up with similar results. One should not forget that one has to normalize Green functions in a manner similar to what we did for Yukawa theory. Table(4) gives the results of Table(1) for the QED case. Considering the vertex correction at zmt, we are confronted with $\bar{\Gamma}_{\mu}^{[1]}(i)$, which is defined in the obvious manner. We then allow for a varying number of $i_{1}$ one-loop subdivergences in the fermion line and $i_{2}$ subdivergences in the photon line, $i=i_{1}+i_{2}$. A moment of thinking ensures that part of the problem reduces to the concatenation of $G$-functions that we have already considered for Yukawa theory. But QED is much more demanding: inserting the $k_{\mu} k_{\nu}$ part of the boson propagator, one is also confronted with $G$ functions which have intermediate IR divergences, as for example

$$
\int d^{D} k \frac{1}{\left[k^{2}\right]^{2}\left[(k+q)^{2}\right]^{j \epsilon}} .
$$

\footnotetext{
${ }^{7}$ Usually, memory and CPU time restrictions allowed for tests up to 10-20 loops.
} 
$\Gamma_{2}(1):=2 / 3 \epsilon^{(-1)}-1 / 2 \epsilon^{(-2)}+1 / 6 \epsilon^{(-3)}$

$\Gamma_{2}(2):=\epsilon^{(-1)}(1 / 80 \zeta(4)+4 / 15)-2 / 15 \epsilon^{(-2)}+17 / 60 \epsilon^{(-3)}-1 / 5 \epsilon^{(-4)}+1 / 20 \epsilon^{(-5)}$

$\overline{\Gamma_{2}(3):=\epsilon^{(-1)}\left(-47 / 336 \zeta(6)+11 / 56 \zeta(5)+3 / 140 \zeta(4)+13 / 70 \zeta(3)^{2}+9 / 70 \zeta(3)+2 / 7\right)+\epsilon^{(-2)}(-11 / 280 \zeta(5)-}$ $3 / 28 \zeta(4)+1 / 70 \zeta(3)+1 / 35)+\epsilon^{(-3)}(3 / 140 \zeta(4)-1 / 14 \zeta(3)+1 / 14)+\epsilon^{(-4)}(1 / 70 \zeta(3)-39 / 280)+47 / 280 \epsilon^{(-5)}-$ $5 / 56 \epsilon^{(-6)}+1 / 56 \epsilon^{(-7)}$

$\bar{\Gamma}_{2}(4):=\epsilon^{(-1)}\left(-575 / 1152 \zeta(8)+167 / 168 \zeta(7)-137 / 216 \zeta(6)+18 / 35 \zeta(5) \zeta(3)+18 / 35 \zeta(5)+13 / 140 \zeta(4)^{2}-\right.$ $\left.26 / 35 \zeta(4) \zeta(3)-17 / 84 \zeta(4)+286 / 315 \zeta(3)^{2}+2 / 315 \zeta(3)+136 / 315\right)+\epsilon^{(-2)}(-167 / 1008 \zeta(7)+113 / 1008 \zeta(6)-$ $\left.6 / 35 \zeta(5)+13 / 105 \zeta(4) \zeta(3)-19 / 210 \zeta(4)-26 / 105 \zeta(3)^{2}-17 / 126 \zeta(3)+4 / 45\right)+\epsilon^{(-3)}(-113 / 6048 \zeta(6)+$ $\left.2 / 15 \zeta(4)+13 / 315 \zeta(3)^{2}-19 / 315 \zeta(3)+2 / 63\right)+\epsilon^{(-4)}(-1 / 14 \zeta(4)+4 / 45 \zeta(3)-1 / 63)+\epsilon^{(-5)}(1 / 84 \zeta(4)-$ $1 / 21 \zeta(3)+377 / 5040)+\epsilon^{(-6)}(1 / 126 \zeta(3)-59 / 504)+25 / 252 \epsilon^{(-7)}-1 / 24 \epsilon^{(-8)}+1 / 144 \epsilon^{(-9)}$

$\bar{\Gamma}_{2}(5):=\quad \epsilon^{(-1)}(-5199 / 3520 \zeta(10)+11333 / 3168 \quad \zeta(9)-45701 / 12672 \quad \zeta(8)+565 / 616 \quad \zeta(7) \zeta(3)+$ $28505 / 11088 \zeta(7)+25 / 56 \zeta(6) \zeta(4)-25 / 12 \zeta(6) \zeta(3)-65 / 63 \zeta(6)+43 / 88 \zeta(5)^{2}-133 / 88 \zeta(5) \zeta(4)+$ $860 / 231 \zeta(5) \zeta(3)+631 / 1848 \zeta(5)+53 / 77 \zeta(4)^{2}-135 / 616 \zeta(4) \zeta(3)^{2}-1667 / 924 \zeta(4) \zeta(3)-47 / 168 \zeta(4)+$ $\left.15 / 44 \zeta(3)^{3}+6617 / 5544 \zeta(3)^{2}-485 / 1386 \zeta(3)+538 / 693\right)+\epsilon^{(-2)}(-1619 / 3168 \zeta(9)+2737 / 2816 \zeta(8)-$ $1835 / 1584 \zeta(7)+25 / 84 \zeta(6) \zeta(3)+25 / 63 \zeta(6)+19 / 88 \zeta(5) \zeta(4)-133 / 132 \zeta(5) \zeta(3)-701 / 1848 \zeta(5)-$ $\left.69 / 352 \zeta(4)^{2}+212 / 231 \zeta(4) \zeta(3)+15 / 1232 \zeta(4)-15 / 308 \zeta(3)^{3}-1667 / 2772 \zeta(3)^{2}-47 / 252 \zeta(3)+19 / 99\right)+$ $\epsilon^{(-3)}\left(-391 / 2816 \zeta(8)+305 / 1056 \zeta(7)-25 / 252 \zeta(6)+19 / 132 \zeta(5) \zeta(3)+3 / 44 \zeta(5)+69 / 2464 \zeta(4)^{2}-\right.$ $\left.23 / 88 \zeta(4) \zeta(3)+185 / 3696 \zeta(4)+212 / 693 \zeta(3)^{2}+5 / 616 \zeta(3)+1 / 18\right)+\epsilon^{(-4)}(-305 / 7392 \zeta(7)+1 / 33 \zeta(5)+$ $\left.23 / 616 \zeta(4) \zeta(3)-251 / 1848 \zeta(4)-23 / 264 \zeta(3)^{2}+185 / 5544 \zeta(3)+37 / 2772\right)+\epsilon^{(-5)}(-7 / 264 \zeta(5)+$ $\left.97 / 924 \zeta(4)+23 / 1848 \zeta(3)^{2}-251 / 2772 \zeta(3)+1 / 72\right)+\epsilon^{(-6)}(1 / 264 \zeta(5)-7 / 176 \zeta(4)+97 / 1386 \zeta(3)-$ $263 / 7392)+\epsilon^{(-7)}(1 / 176 \zeta(4)-7 / 264 \zeta(3)+1699 / 22176)+\epsilon^{(-8)}(1 / 264 \zeta(3)-139 / 1584)+91 / 1584 \epsilon^{(-9)}-$ $7 / 352 \epsilon^{(-10)}+1 / 352 \epsilon^{(-11)}$

Table 3: Two-loop rainbow dressings. 


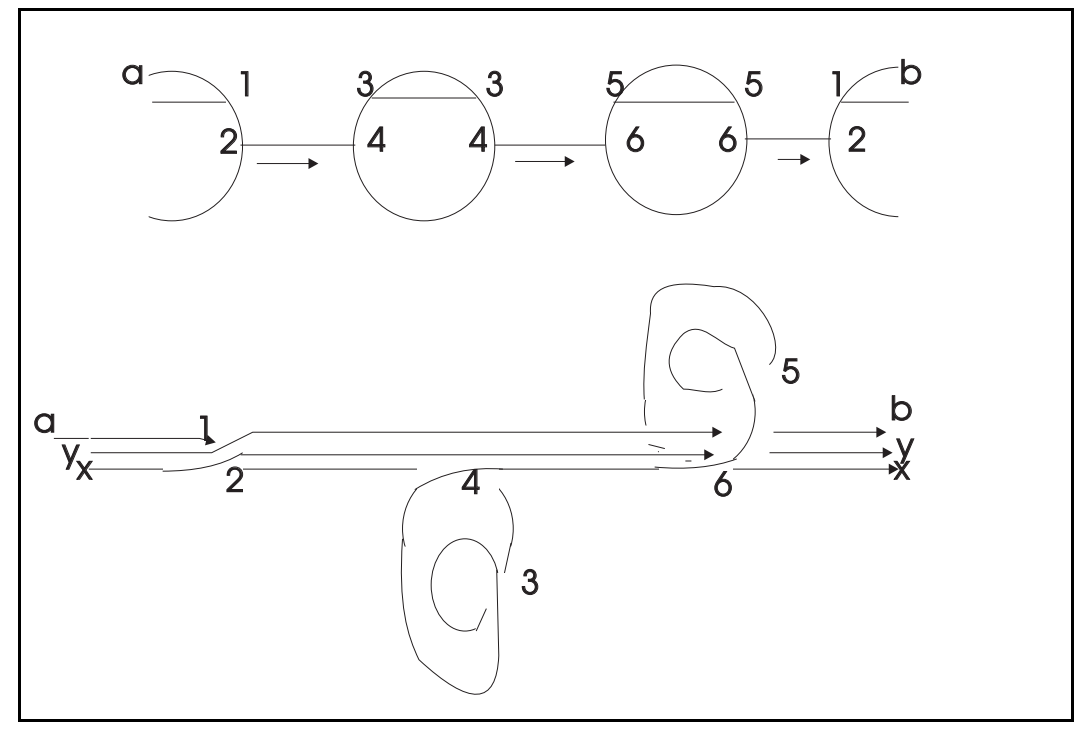

Figure 9: One further cabling of the strand from $a$ to $b$ produces a knot whose presence matches the transcendentals in Table(3), here demonstrated for $\bar{\Gamma}_{2}(3)$. The knot is obtained by the identifications at $x, y$ and $a$ with $b$, and turns out to be the $(2,5)$ torus knot.

\begin{tabular}{l}
$\overline{\bar{\Gamma}_{Q E D}(1):=4 \epsilon^{(-1)}-3 \epsilon^{(-2)}}$ \\
$\overline{\bar{\Gamma}}_{Q E D}(2):=-4 / 3 \epsilon^{(-1)}-8 / 3 \epsilon^{(-2)}+2 \epsilon^{(-3)}$ \\
$\overline{\bar{\Gamma}}_{Q E D}(3):=\epsilon^{(-1)}(-3 \zeta(3)+1)+\epsilon^{(-2)}+2 \epsilon^{(-3)}-3 / 2 \epsilon^{(-4)}$ \\
$\overline{\bar{\Gamma}}_{Q E D}(4):=\epsilon^{(-1)}(18 / 5 \zeta(4)-16 / 5 \zeta(3)-4 / 5)+\epsilon^{(-2)}(12 / 5 \zeta(3)-4 / 5)-4 / 5 \epsilon^{(-3)}-8 / 5 \epsilon^{(-4)}+6 / 5 \epsilon^{(-5)}$ \\
\hline
\end{tabular}

Table 4: Two- to five-loop QED examples.

In Table(4) we only show the results for the part of the vertex which was infected by such $G$-functions, and omit the contributions which were similar to the Yukawa case from the start. Again counterterms are

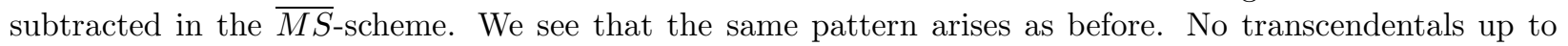
three loops, and then $\zeta(3)$ plus rational contributions at four loops.

Finally, let us comment on two results obtained by other authors. In both cases, calculations were pushed to the four-loop level, while investigating $\beta$-functions of some sort.

First, the investigation of $\phi^{4}$ theory in 19 considers the four-loop level. The four-loop result is in precise agreement with our expectations. Again, $\zeta(3)$ appears at the four-loop level, from diagrams where we expect it to occur. In fact, the circle chain integral (as the author calls it) $I_{3}^{c c}$ is topologically equivalent to our four-loop graph in the first example, and thus we expect the author to find a contribution containing $\zeta(3)$ as well as a rational part, which is indeed the case.

Second, in [20], it is conjectured that the appearance of $\zeta(3)$ at only three places out of twelve different topologies (cf. Fig. 4 in the paper) could be explained using knot theory. With the experience from the results presented here, this is indeed the case. The three cases (Fig.4F,G,H in the paper) which deliver $\zeta(3)$ in the results of [20] are again topologically equivalent to the four-loop case in our first example, while all the other 
diagrams only provide link diagrams free of knots, as they should. In our notation, they all belong to cases similar to $\bar{\Gamma}_{n}(1,0)$.

\section{Conclusions}

In this paper we compared overall divergences of Feynman graphs containing subdivergences with link diagrams. Using recent results on a connection between knot theory and renormalization theory, we explored the appearance of transcendentals in the overall divergences. Our results indicate that also graphs which do contain subdivergences can be investigated using link and knot theory. This closes the before-mentioned gap between results concerning topologically trivial ladder graphs and results concerning graphs of complicated topology, but free of subdivergences. In this paper we considered the middle ground inbetween.

Specifically, we found that disjoint subdivergences of a simple structure do provide transcendentals in accordance with link diagrams assigned to them in the manner proposed in 1, 4, 6, 7]. In the most simple case, we dressed a one-loop skeleton graph with chains of one-loop subdivergences. Link diagrams for this case suggested the appearance of all $(2, q)$ torus knots, with the highest $q$ determined by the loop number. Table(11) summarizes these results.

Using only very basic properties of link diagrams, we discovered a new relation between rational parts of overall counterterms, as it is dramatically exemplified in Table(2). All the Feynman diagrams considered there contain knot-free link diagrams, but some contain others link diagrams as well. We found that whenever a knot-free link diagram results from various different diagrams, the rational part of their contribution to the overall divergence is the same. This not only confirms that knots should be associated with transcendentals, but establishes new relations between diagrams. Apart from our approach via link theory, there is no other explanation for such results available. It indicates that the resolution of the topology of a Feynman diagram in terms of different link diagrams is a meaningful tool to understand the number-theoretic properties of counterterms. The way we assign knots of increasing complexity to these diagrams is by way of a cabling operation, which indicates further systematics in this approach which deserves further study.

These relations between diagrams were not found before, mainly, one guesses, because one barely calculates overall divergent quantities by doing the renormalization of subdivergences graph by graph. Multiplicative renormalization screens these new findings. Only when one renormalizes graph by graph, one can observe these relations.

It seems that a reorganization of perturbative results ordered with respect to transcendentals is favourable. Our results indicate that the forest structure of a graph has a deep connection to link theory, where the way how forests are mutually disjoint or nested is reflected in the entanglement of link diagrams which one can assign to them.

Further results confirmed our expectations, and we also gave some results for QED. We expect that the patterns observed here are true for a renormalizable theory in general. 8

While already results for diagrams without subdivergences deliver evidence towards a connection between the theory of links and knots, number theory, and the problem of divergences in a pQFT, it is apparent that the full richness of this new connection arises when one allows for the general case. In this new area, much remains to be done, and the results reported here are only the first few steps towards an understanding of this connection. It still seems that the role which UV divergences of a quantum field theory play is not fully explored yet. Here is not the space to muse about connections to recent developments in mathematics, and we refer to [18] for such purposes.

In recent results, 9, 10], results are obtained clarifying the role of the four-term relation in counterterms free of subdivergences. These results still suffer from an exclusion of graphs with subdivergences. In the light of the results here we hope to be able to report on some progress with the four-term relation concerning graphs with subdivergences soon.

\footnotetext{
${ }^{8}$ At least in an even dimensional field theory. In odd dimensions, one might find a different set of transcendentals. I thank D. Broadhurst for stressing this point.
} 


\section{Acknowledgements}

Foremost, I have to thank David Broadhurst for the enthusiam and vigor with which he picked up these new ideas and turned them into a most fruitful collaboration. With equal pleasure I thank Bob Delbourgo for uncounted discussions and support. Also, I like to thank Karl Schilcher and Jürgen Körner for interest and encouragement, and the DFG for support.

\section{References}

[1] D. Kreimer, Habilschrift: Renormalization and Knot Theory, to appear in J.Knot Th.Ram., MZ-TH/96$18, q-\operatorname{alg} / 9607022$.

[2] D.J. Broadhurst, On the enumeration of irreducible $k$-fold Euler sums and their roles in knot theory and field theory, to appear in J.Math.Phys., Open Univ. preprint OUT-4102-62 (1996), hep-th/9604128.

[3] D. Kreimer, Phys.Lett.B354 (1995) 117.

[4] D.J. Broadhurst, D. Kreimer, Int.J.of Mod.Phys.C6 (1995) 519.

[5] D.J. Broadhurst, R. Delbourgo, D. Kreimer, Phys.Lett.B366 (1996) 421.

[6] D.J. Broadhurst, J.A. Gracey, D. Kreimer, Beyond the triangle and uniqueness relations: non-zeta terms at large $N$ from positive knots, to appear in Z.Phys.C, OUT-4102-46, MZ-TH/95-28, hep-th/9607174; D.J. Broadhurst, A. Kotikov, Compact analytical form for non-zeta terms in critical exponents at order $1 / N^{3}$, subm. to Phys.Lett.B, hep-th/9612013.

[7] D.J. Broadhurst, D. Kreimer, Phys.Lett.B393 (1997) 403.

[8] J.M. Borwein, D.A. Bradley, D.J. Broadhurst, Electronic J. Combinatorics 4 (1997) R5.

[9] D. Kreimer, Weight Systems from Feynman Diagrams, subm. to J.Knot Th.Ram., hep-th/9612010.

[10] D.J. Broadhurst, D. Kreimer, Feynman Diagrams as a weight system: four-loop test of a four-term relation, subm. to Phys.Lett.B, hep-th/9612011.

[11] D.J. Broadhurst, Conjectured enumeration of irreducible multiple zeta values, from knots and Feynman diagrams, subm. to Phys.Lett.B, hep-th/9612012.

[12] R. Delbourgo, A. Kalloniatis, G. Thompson, Phys.Rev.D54 (1996) 5373;

R. Delbourgo, D. Elliott, D.S. McAnally, Univ. of Tasmania preprint UTAS-PHYS-96-06, hepth/9611150, to appear in Phys.Rev.D.

[13] D.J. Broadhurst, Massless scalar Feynman diagrams: five loops and beyond, Open Univ. preprint OUT4102-18 (1985).

[14] D. Zagier, in Proc.First European Congress Math. (Birkhäuser, Boston, 1994) Vol.II, pp 497-512; Multiple Zeta Values, in preparation.

[15] K.G. Chetyrkin, A.L. Kataev, F.V. Tkachov, Nucl.Phys.B174 (1980) 345.

[16] W. Zimmermann, Comm.Math.Phys.15 (1969) 208.

[17] L. Kauffman, Knots and Physics, 2nd edition, World Scientific 1993.

[18] D. Kreimer, Knots and Feynman Diagrams (Cambridge UP, in preparation).

[19] B. Kastening, Phys.Rev.D54 (1996) 3965.

[20] I. Jack, D.R.T. Jones, C.G. North, Scheme dependence and the NSVZ $\beta$-function, DAMTP Liverpool preprint LTH 381, hep-ph/9609325. 